\title{
Epidemic Model of HIVIAIDS Transmission Dynamics with Different Latent Stages Based on Treatment
}

\author{
Ram Singh ${ }^{1}$, Shoket Ali ${ }^{1}$, Madhu Jain ${ }^{2}$, Rakhee ${ }^{3}$ \\ ${ }^{1}$ Department of Mathematical Sciences, Baba Ghulam Shah Badshah, University, Rajouri, Jammu and Kashmir, India \\ ${ }^{2}$ Department of Mathematics, Indian Institute of Technology Roorkee, Roorkee, Uttrakhand, India \\ ${ }^{3}$ Department of Mathematics, Birla Institute of Technology and Science, Pilani, Rajasthan, India
}

Email address:

singh_ram2008@hotmail.com (R. Singh), shoketali87@gmail.com (S. Ali),drmadhujain.iitr@gmail.com (M. Jain), rakhee@pilani.bits-pilani.ac.in (Rakhee)

\section{To cite this article:}

Ram Singh, Shoket Ali, Madhu Jain, Rakhee. Epidemic Model of HIV/AIDS Transmission Dynamics with Different Latent Stages Based on Treatment. American Journal of Applied Mathematics. Vol. 4, No. 5, 2016, pp. 222-234. doi: 10.11648/j.ajam.20160405.14

Received: August 30, 2016; Accepted: September 23, 2016; Published: October 14, 2016

\begin{abstract}
The mathematical model for analyzing the transmission dynamics of HIV/AIDS epidemic with treatment is studied by considering the three latent compartments for slow, medium and fast progresses of developing the AIDS. By constructing the system of differential equations for the different population groups namely susceptible, three types of latent individuals, symptomatic stage group and full blown AIDS individuals, the mathematical analysis is carried out in order to understand the dynamics of disease spread. By determining the basic reproduction number $\left(R_{0}\right)$, the model examines the two equilibrium points (i) the disease free equilibrium and (ii) the endemic equilibrium. It is established that if $R_{0}<1$, the disease free equilibrium is locally and globally asymptotically stable. The stability of endemic equilibrium has also been discussed.
\end{abstract}

Keywords: Transmission Dynamic, HIV/AIDS, Latent Compartments, Reproduction Number, Stability

\section{Introduction}

HIV/AIDS is one of the fatal diseases, which causes millions of death in both developed and developing nations. More than 35 million people approximately in the worldwide are living with HIV. In 2013, the newly infected persons were reported around 2.1 million which is $38 \%$ less from 2001 . HIV infections among children are also declined by $58 \%$ since 2001. AIDS related deaths have also fallen by $35 \%$ since the peak in 2005, which is a result of availability of Antiretroviral Therapy (ART). Still tuberculosis related deaths in people living with HIV remain the leading cause of death. Since epidemic modeling initially studied by May and Anderson [1], many advance researches have taken place and several important aspects were incorporated by different authors during last three decades. Several epidemiological investigations related to HIV/AIDS infection have been conducted via mathematical models by many researchers [26]. An epidemiological model with nonlinear incident rate was discussed by several authors [7, 8]. Cai et al. [9] established the ordinary differential equation (ODE) model with two infective stages before transition to AIDS. They considered by all sort of treatment methods by considering that some individuals transformed into asymptomatic individuals from symptomatic individuals. The capacity of human immune system can be reduced by chronic diseases, such as diabetes and tuberculosis and can reduce. To analyze this behavior, Huo and Feng [10] developed a model with slow and fast latent compartments. Okosun et al. [11] studied the treatment of HIV/AIDS and screening of unaware infective on the transmission dynamics of disease in a homogeneous population. Defeng and Wang [12] proposed a time delayed mathematical model to analyze the effect of vaccination and ART (Antiretroviral Therapy) on HIV/AIDS. They considered two types of individuals one who are aware of their infected stage and other individuals are unaware about their infected stage. Bhunu and Mushayabasa [13] formulated a mathematical model of the co-dynamics of hepatitis $\mathrm{C}$ virus and HIV/AIDS in order to assess their impact on the dynamics of each disease in the presence of treatment. Cai et al. [14] investigated an HIV/AIDS treatment model with multiple infection stages and treatment where infection was assumed to be of density dependent 
form. Kaur et al. [15] also proposed a nonlinear model for studying the transmission dynamics of HIV/AIDS epidemic with emphasis on the role of female sex workers. Elaiw and Almuallem [16] to investigate the qualitative behaviors of three HIV dynamical models with two types of co circulating target cells. Wang et al. [17] has been studied the global stability of HIV viral infection model with continuous agestructure using the direct lyapunov method. Shen et al. [18] to analyze the mathematical model of global dynamics with two lyapunov functions are constructed to prove the global stability of disease free and endemic equilibria. Treatment class of HIV/AIDS epidemic model is introduced by Huo et al. [19].

In this paper, the mathematical model have been proposed for analyzing the transmission dynamics of HIV/AIDS epidemic with treatment by considering the three latent compartments for slow, medium and fast progresses of developing the AIDS. To study the dynamics of the spread of $\mathrm{HIV}$, the basic reproduction number under disease free equilibrium is analyzed. The global stability of the endemic equilibrium for some special cases is discussed. The remaining contents of the paper are organized into different sections as follows. Section 2 presents the model description, system equations and some basic properties. Stability analysis is done in the section 3. Numerical results to support the proposed model are provided in section 4 . Section 5 summaries the important findings and scope of the future works.

\section{Model Description}

The mathematical model to analyze the transmission dynamic of HIV/AIDS epidemic is developed by dividing the total population into six compartments, namely the susceptible compartment $(S)$, slow latent compartment $\left(I_{1}\right)$, medium compartment $\left(I_{2}\right)$, fast latent compartment $\left(I_{3}\right)$, symptomatic stage $(J)$ and a full-blown AIDS $(A)$ group. The model flow depicting the biological system is illustrated in fig. 1 . The total number of population at time $t$ is given by

$$
N(t)=S(t)+I_{1}(t)+I_{2}(t)+I_{3}(t)+J(t)+A(t) .
$$

For the mathematical formulation of the model, the following notations are used:

$\Lambda$ Recruitment rate of the population

$\beta$ Transmission coefficient of the fast latent compartment $\beta_{2}$ Transmission coefficient of the symptomatic stage $p_{1}, p_{2}$ The fraction of susceptible $S$ being infected by $I_{3}$ and entering into $I_{1}, I_{2}$, respectively; $p_{3}=1-p_{1}-p_{2}$ $q_{1}, q_{2}$ The fraction of susceptible $S$ being infected by $J$ and entering into $I_{1}, I_{2}$, respectively; $q_{3}=1-q_{1}-q_{2}$

$\varepsilon_{i}$ Progression rate from latent compartment $I_{i}$ to $I_{i+1} ; i=1,2$

$r$ Progression rate from latent compartment $I_{3}$ to symptomatic compartment $J$

$\rho$ Progression rate of disease from compartment $J$ to $A$

$\xi_{i}$ Treatment rate from compartment $J$ to $I_{i} ; i=1,2,3$

$\mu(\alpha)$ Natural (disease induced) death rate

\subsection{The Governing Equations}

The following differential equations governing the model are constructed by considering the appropriate in-flow and out-flow rates of each compartment:

$$
\begin{gathered}
\frac{d S(t)}{d t}=\Lambda-\left(p_{1}+p_{2}+p_{3}\right) \beta_{1} I_{3}(t) S(t)-\left(q_{1}+q_{2}+q_{3}\right) \beta_{2} J(t) S(t)-\mu S(t) \\
\frac{d I_{1}(t)}{d t}=p_{1} \beta_{1} I_{3}(t) S(t)+q_{1} \beta_{2} J(t) S(t)-\left(\varepsilon_{1}+\mu\right) I_{1}(t)+\xi_{1} J(t) \\
\frac{d I_{2}(t)}{d t}=p_{2} \beta_{1} I_{3}(t) S(t)+q_{2} \beta_{2} J(t) S(t)+\varepsilon_{1} I_{1}(t)-\left(\varepsilon_{2}+\mu\right) I_{2}(t)+\xi_{2} J(t) \\
\frac{d I_{3}(t)}{d t}=p_{3} \beta_{1} I_{3}(t) S(t)+q_{3} \beta_{2} J(t) S(t)+\varepsilon_{2} I_{2}(t)-(r+\mu) I_{3}(t)+\xi_{3} J(t) \\
\frac{d J(t)}{d t}=r I_{3}(t)-\left(\xi_{1}+\xi_{2}+\xi_{3}+\rho+\mu\right) J(t) \\
\frac{d A(t)}{d t}=\rho J(t)-(\mu+\alpha) A(t)
\end{gathered}
$$

For brevity of notation, by using

$$
b_{1}=\varepsilon_{1}+\mu, b_{2}=\varepsilon_{2}+\mu, b_{3}=r+\mu, b_{4}=\xi_{1}+\xi_{2}+\xi_{3}+\rho+\mu, \text { and } b_{5}=\mu+\alpha
$$


The above set of equations (1)-(6) becomes

$$
\begin{gathered}
\frac{d S(t)}{d t}=\Lambda-\beta_{1} I_{3}(t) S(t)-\beta_{2} J(t) S(t)-\mu S(t) \\
\frac{d I_{1}(t)}{d t}=p_{1} \beta_{1} I_{3}(t) S(t)+q_{1} \beta_{2} J(t) S(t)-b_{1} I_{1}(t)+\xi_{1} J(t) \\
\frac{d I_{2}(t)}{d t}=p_{2} \beta_{1} I_{3}(t) S(t)+q_{2} \beta_{2} J(t) S(t)+\varepsilon_{1} I_{1}(t)-b_{2} I_{2}(t)+\xi_{2} J(t) \\
\frac{d I_{3}(t)}{d t}=p_{3} \beta_{1} I_{3}(t) S(t)+q_{3} \beta_{2} J(t) S(t)+\varepsilon_{2} I_{2}(t)-b_{3} I_{3}(t)+\xi_{3} J(t) \\
\frac{d J(t)}{d t}=r I_{3}(t)-b_{4} J(t) \\
\frac{d A(t)}{d t}=\rho J(t)-b_{5} A(t)
\end{gathered}
$$

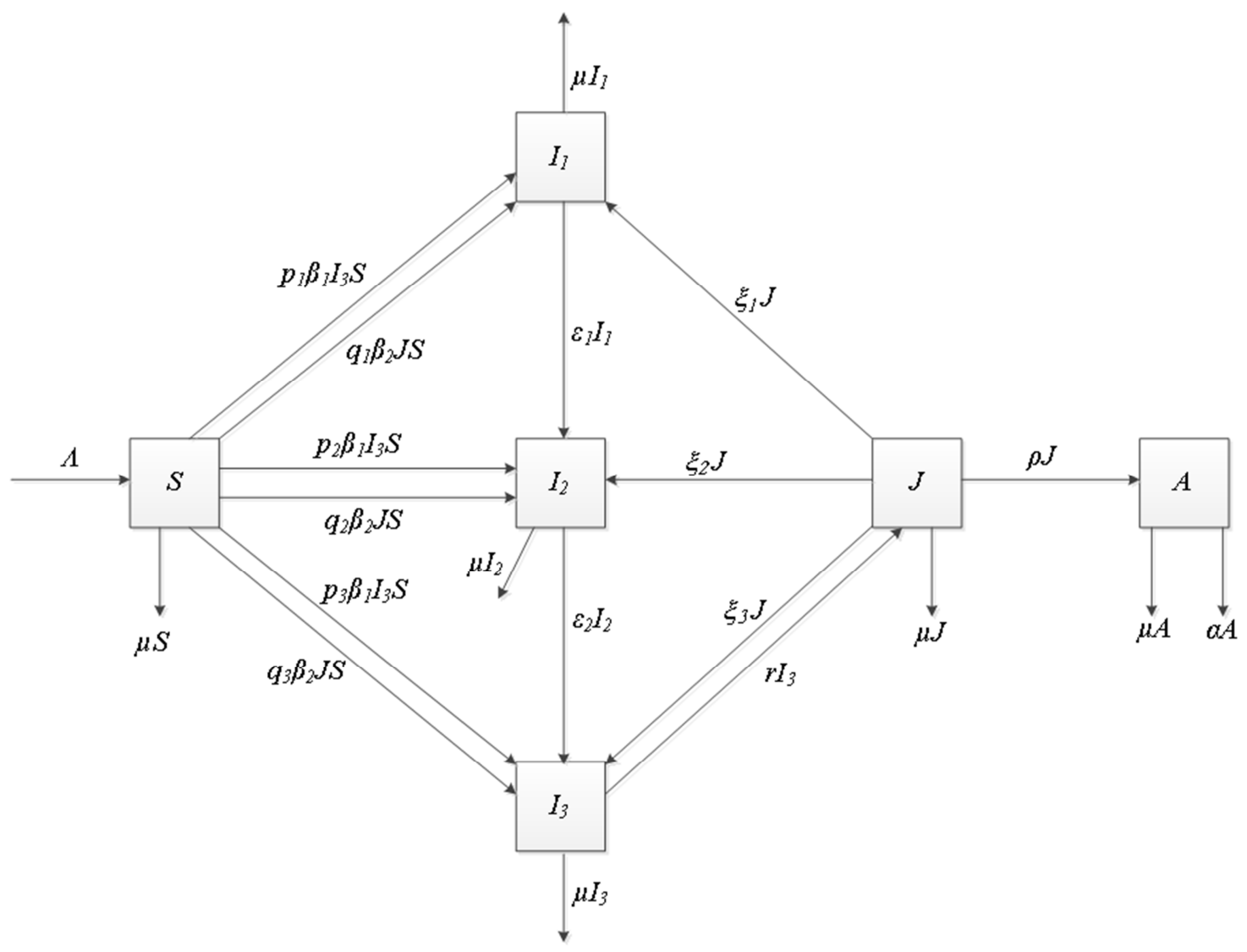

Fig. 1. Transmission dynamic diagram.

\subsection{Basic Properties}

In this sub-section, we discuss some preliminary concepts which will be needed further for the mathematical analysis of the concerned model. We will show that for $t \geq 0$, all the solutions are positively invariant in some region.

\subsubsection{Invariant Region}

Since the model displays the changes in the human population, the variables and the parameters are assumed to be positive for all $t \geq 0$. The system of equations (7)-(12) will therefore be analyzed in a suitable feasible region $\Omega$ of biological interest. We have the following lemma related to feasible region for the system (7)-(12). 
Lemma1: The feasible region $\Omega$ defined by

$$
\Omega=\left\{\left(S(t), I_{1}(t), I_{2}(t), I_{3}(t), J(t), A(t)\right) \in R_{+}^{6}: N(t) \leq \frac{\Lambda}{\mu}\right\}
$$

with initial conditions

$S(0) \geq 0, I_{1}(0) \geq 0, I_{2}(0) \geq 0, I_{3}(0) \geq 0, J(0) \geq 0, A(0) \geq 0$ is positively invariant for the system of equations (7)-(12).

Proof: Adding the system of equations (1)-(6), we obtain

$$
\frac{d N}{d t}=\Lambda-\mu N-\alpha A \leq \Lambda-\mu N
$$

Solving the above differential equation (13), we have

$$
0 \leq N(t) \leq \frac{\Lambda}{\mu}+N(0) e^{-\mu t}
$$

where $N(0)$ represents the initial values of the total population. Thus $\lim _{t \rightarrow+\infty} \sup N(t) \leq \frac{\Lambda}{\mu}$.

(i) there exists a first time $t_{1}$ such that

$$
S\left(t_{1}\right)=0, S^{\prime}\left(t_{1}\right)<0, I_{1}(t) \geq 0, I_{2}(t) \geq 0, I_{3}(t) \geq 0, J(t) \geq 0, A(t) \geq 0,0 \leq t \leq t_{1},
$$

(ii) there exists a $t_{2}$

$$
I_{1}\left(t_{2}\right)=0, I_{1}^{\prime}\left(t_{2}\right)<0, S(t) \geq 0, I_{2}(t) \geq 0, I_{3}(t) \geq 0, J(t) \geq 0, A(t) \geq 0,0 \leq t \leq t_{2},
$$

(iii) there exists a $t_{3}$

$$
I_{2}\left(t_{3}\right)=0, I_{2}^{\prime}\left(t_{3}\right)<0, S(t) \geq 0, I_{1}(t) \geq 0, I_{3}(t) \geq 0, J(t) \geq 0, A(t) \geq 0,0 \leq t \leq t_{3},
$$

(iv) there exists a $t_{4}$

$$
I_{3}\left(t_{4}\right)=0, I_{3}^{\prime}\left(t_{4}\right)<0, S(t) \geq 0, I_{1}(t) \geq 0, I_{2}(t) \geq 0, J(t) \geq 0, A(t) \geq 0,0 \leq t \leq t_{4},
$$

(v) there exists a $t_{5}$

$$
J\left(t_{5}\right)=0, J^{\prime}\left(t_{5}\right)<0, S(t) \geq 0, I_{1}(t) \geq 0, I_{2}(t) \geq 0, I_{3}(t) \geq 0, A(t) \geq 0,0 \leq t \leq t_{5},
$$

(vi) there exists a $t_{6}$

$$
A\left(t_{6}\right)=0, A^{\prime}\left(t_{6}\right)<0, S(t) \geq 0, I_{1}(t) \geq 0, I_{2}(t) \geq 0, I_{3}(t) \geq 0, J(t) \geq 0,0 \leq t \leq t_{6},
$$

In the first case (i), we have

$$
S^{\prime}\left(t_{1}\right)=\Lambda>0
$$

which is a contradiction meaning that $S(t) \geq 0, t \geq 0$.

In the second case (ii), we have

$$
I_{1}^{\prime}\left(t_{2}\right)=p_{1} \beta_{1} I_{3}\left(t_{2}\right) S\left(t_{2}\right)+q_{1} \beta_{2} J\left(t_{2}\right) S\left(t_{2}\right)+\xi_{1} J\left(t_{2}\right) \geq 0
$$

which is a contradiction meaning that $I_{1}(t) \geq 0, t \geq 0$. Similarly, from (iii)-(vi), it can be easily shown that $I_{2}(0)>$ $0, I_{3}(0)>0, J(0)>0$, and $A(0)>0$, for all $t \geq 0$.Thus, the solutions $S(t), I_{1}(t), I_{2}(t), I_{3}(t), J(t)$ and $A(t)$ of system (7)(12) remain positive for all $t>0$.

\section{The Analysis}

In this section, we compute the equilibrium states, namely the disease free equilibrium (DFE) and the endemic equilibrium (EE) and provide stability analysis by determining the basic reproduction number.

\subsection{Disease Free Equilibrium (DFE) and Basic Reproduction Number $\boldsymbol{R}_{0}$.}


Proposed model (7)-(12) has a disease free equilibrium given by

$$
E_{0}=\left(S^{0}, I_{1}^{0}, I_{2}^{0}, I_{3}^{0}, J^{0}, A^{0}\right)=\left(\frac{\Lambda}{\mu}, 0,0,0,0,0\right)
$$

The basic reproduction number of the system (7)-(12) is obtained by considering the next generation matrices $F$ and $V$ defined for the appearance of new infection terms and the transfer of individuals out of latent compartment, respectively [20]. Let $X=\left(I_{1}, I_{2}, I_{3}, J, A, S\right)^{T}$, then the system (7)-(12) can be written as

$$
\frac{d X}{d t}=F(X)-V(X)
$$

where

$$
F(X)=\left[\begin{array}{c}
p_{1} \beta_{1} I_{3} S+q_{1} \beta_{2} J S \\
p_{2} \beta_{1} I_{3} S+q_{2} \beta_{2} J S \\
p_{3} \beta_{1} I_{3} S+q_{3} \beta_{2} J S \\
0 \\
0 \\
0
\end{array}\right]
$$

and

$$
V(X)=\left[\begin{array}{l}
b_{1} I_{1}-\xi_{1} J \\
b_{2} I_{2}-\xi_{2} J-\varepsilon_{1} I_{1} \\
b_{3} I_{3}-\xi_{3} J-\varepsilon_{2} I_{2} \\
b_{4} J-r I_{3} \\
b_{5} A-\rho J \\
\beta_{1} I_{3} S+\beta_{2} J S+\mu S-\Lambda
\end{array}\right]
$$

Now we employ the linearization method. On taking partial derivatives, the associated matrices at DFE are obtained as,

$$
F=\left[\begin{array}{ccccc}
0 & 0 & p_{1} \beta_{1} \frac{\Lambda}{\mu} & q_{1} \beta_{2} \frac{\Lambda}{\mu} & 0 \\
0 & 0 & p_{2} \beta_{1} \frac{\Lambda}{\mu} & q_{2} \beta_{2} \frac{\Lambda}{\mu} & 0 \\
0 & 0 & p_{3} \beta_{1} \frac{\Lambda}{\mu} & q_{3} \beta_{2} \frac{\Lambda}{\mu} & 0 \\
0 & 0 & 0 & 0 & 0 \\
0 & 0 & 0 & 0 & 0
\end{array}\right]
$$

and

$$
V=\left[\begin{array}{ccccc}
b_{1} & 0 & 0 & -\xi_{1} & 0 \\
-\varepsilon_{1} & b_{2} & 0 & -\xi_{2} & 0 \\
0 & -\varepsilon_{2} & b_{3} & -\xi_{3} & 0 \\
0 & 0 & -r & b_{4} & 0 \\
0 & 0 & 0 & -\rho & b_{5}
\end{array}\right]
$$

Consider the following matrix

$$
F V^{-1}=\left[\begin{array}{ccccc}
A_{1} & B_{1} & C_{1} & D_{1} & 0 \\
A_{2} & B_{2} & C_{2} & D_{2} & 0 \\
A_{3} & B_{3} & C_{3} & D_{3} & 0 \\
0 & 0 & 0 & 0 & 0 \\
0 & 0 & 0 & 0 & 0
\end{array}\right]
$$

where

$$
\begin{aligned}
& A_{i}=\left[\frac{p_{i} \beta_{1} b_{4} \varepsilon_{1} \varepsilon_{2}+q_{i} \beta_{2} \varepsilon_{1} \varepsilon_{2} r}{W}\right] \frac{\Lambda}{\mu} ; i=1,2,3 \\
& B_{i}=\left[\frac{p_{i} \beta_{1} b_{1} b_{4} \varepsilon_{2}+q_{i} \beta_{2} b_{1} \varepsilon_{2} r}{W}\right] \frac{\Lambda}{\mu} ; i=1,2,3 \\
& C_{i}=\left[\frac{p_{i} \beta_{1} b_{1} b_{2} b_{4}+q_{i} \beta_{2} b_{1} b_{2} r}{W}\right] \frac{\Lambda}{\mu} ; i=1,2,3
\end{aligned}
$$

$$
\begin{gathered}
D_{i}=\left[\frac{p_{i} \beta_{1}\left(b_{1} b_{2} \xi_{3}+b_{1} \varepsilon_{2} \xi_{2}+\varepsilon_{1} \varepsilon_{2} \xi_{1}\right)+q_{i} \beta_{2} b_{1} b_{2} b_{3}}{W}\right] \frac{\Lambda}{\mu} ; i=1,2,3 \\
W=b_{1} b_{2} b_{3} b_{4}-b_{1} b_{2} \xi_{3} r-b_{1} \varepsilon_{2} \xi_{2} r-\varepsilon_{1} \varepsilon_{2} \xi_{1} r
\end{gathered}
$$

Thus the reproduction number $R_{0}$, is obtained as

$$
R_{0}=\rho\left(F V^{-1}\right)=\left[\frac{\left(p_{1} \beta_{1} b_{4}+q_{1} \beta_{2} r\right) \varepsilon_{1} \varepsilon_{2}+\left(p_{2} \beta_{1} b_{4}+q_{2} \beta_{2} r\right) b_{1} \varepsilon_{2}+\left(p_{3} \beta_{1} b_{4}+q_{3} \beta_{2} r\right) b_{1} b_{2}}{b_{1} b_{2}\left(b_{3} b_{4}-\xi_{3} r\right)-\varepsilon_{2} r\left(b_{1} \xi_{2}-\varepsilon_{1} \xi_{1}\right)}\right] \frac{\Lambda}{\mu}
$$

Following Theorem 2 of Van den Driessche and Watmough [17], we have the following result on the local stability of $E_{0}$ : Theorem 1: The disease free equilibrium $E_{0}$ of the system (7)-(12) is locally asymptotically stable if $R_{0}<1$ and unstable if $R_{0}>1$.

\subsection{Global Stability of Disease Free Equilibrium (DFE)}

Here we analyze the global stability by using a comparison theorem [21, 22].

Theorem 2: The disease free equilibrium $E_{0}$ of the system 
(7)-(12) is globally asymptotically stable if $R_{0}<1$ and unstable if $R_{0}>1$.
Proof: The equation of the infected components in system (7)-(12) can be written as

$$
\left[\begin{array}{l}
I_{1}^{\prime} \\
I_{2}^{\prime} \\
I_{3}^{\prime} \\
J^{\prime} \\
A^{\prime}
\end{array}\right]=[F-V]\left[\begin{array}{l}
I_{1} \\
I_{2} \\
I_{3} \\
J \\
A
\end{array}\right]-\left[1-\frac{\mu}{\Lambda} S\right]\left[\begin{array}{ccccc}
0 & 0 & p_{1} \beta_{1} \frac{\Lambda}{\mu} & q_{1} \beta_{2} \frac{\Lambda}{\mu} & 0 \\
0 & 0 & p_{2} \beta_{1} \frac{\Lambda}{\mu} & q_{2} \beta_{2} \frac{\Lambda}{\mu} & 0 \\
0 & 0 & p_{3} \beta_{1} \frac{\Lambda}{\mu} & q_{3} \beta_{2} \frac{\Lambda}{\mu} & 0 \\
0 & 0 & 0 & 0 & 0 \\
0 & 0 & 0 & 0 & 0
\end{array}\right]\left[\begin{array}{l}
I_{1} \\
I_{2} \\
I_{3} \\
J \\
A
\end{array}\right]
$$

where $\mathrm{F}$ and $\mathrm{V}$ are defined in (17) and (18) for all $t \geq 0$ in

$\Omega$.

Thus, we have

$$
\left[\begin{array}{l}
I_{1}^{\prime} \\
I_{2}^{\prime} \\
I_{3}^{\prime} \\
J^{\prime} \\
A^{\prime}
\end{array}\right] \leq[F-V]\left[\begin{array}{l}
I_{1} \\
I_{2} \\
I_{3} \\
J \\
A
\end{array}\right]
$$

Since all eigen values of the matrix $F-V$ have negative real parts, the linearized differential inequality (22) is stable whenever $R_{0}<1$.

Consequently $\left(I_{1}, I_{2}, I_{3}, J, A\right) \rightarrow(0,0,0,0,0)$ as $t \rightarrow \infty$. Thus following the comparison theorem, we have $\left(I_{1}, I_{2}, I_{3}, J, A\right) \rightarrow(0,0,0,0,0)$ and $S \rightarrow \frac{\Lambda}{\mu}$ as $t \rightarrow \infty$.

Hence the $\operatorname{DFE}\left(E_{0}\right)$ is globally asymptotically stable for $R_{0}<1$.

\subsection{Endemic Equilibrium (EE)}

The endemic equilibrium point of the system (7)-(12) is given

$$
V_{1}=\left(S-S^{*} \ln S\right)+B\left(I_{1}-I_{1}^{*} \ln I_{1}\right)+C\left(I_{2}-I_{2}^{*} \ln I_{2}\right)+D\left(I_{3}-I_{3}^{*} \ln I_{3}\right)+E\left(J-J^{*} \ln J\right)
$$

The derivative of $V_{1}$ is

$$
\begin{aligned}
& \quad V_{1}^{\prime}=\left(1-\frac{S^{*}}{S}\right) S^{\prime}+B\left(1-\frac{I_{1}^{*}}{I_{1}}\right) I_{1}^{\prime}+C\left(1-\frac{I_{2}^{*}}{I_{2}}\right) I_{2}^{\prime}+D\left(1-\frac{I_{3}^{*}}{I_{3}}\right) I_{3}^{\prime}+E\left(1-\frac{J^{*}}{J}\right) J^{\prime} \\
& =\left(1-\frac{S^{*}}{S}\right)\left[\Lambda-\left(\beta_{1} I_{3} S+\beta_{2} J S\right)-\mu S\right]+B\left(1-\frac{I_{1}^{*}}{I_{1}}\right)\left[p_{1} \beta_{1} I_{3} S+q_{1} \beta_{2} J S+\xi_{1} J-b_{1} I_{1}\right] \\
& +C\left(1-\frac{I_{2}^{*}}{I_{2}}\right)\left[p_{2} \beta_{1} I_{3} S+q_{2} \beta_{2} J S+\varepsilon_{1} I_{1}+\xi_{2} J-b_{2} I_{2}\right] \\
& +D\left(1-\frac{I_{3}^{*}}{I_{3}}\right)\left[p_{3} \beta_{1} I_{3} S+q_{3} \beta_{2} J S+\varepsilon_{2} I_{2}+\xi_{3} J-b_{3} I_{3}\right]+E\left(1-\frac{J^{*}}{J}\right)\left[r I_{3}-b_{4} J\right]
\end{aligned}
$$


The system (7)-(12) satisfies the following relation at the equilibrium point:

$$
\begin{gathered}
\Lambda=\beta_{1} I_{3}^{*} S^{*}+\beta_{2} J^{*} S^{*}+\mu S^{*} \\
b_{1}=\frac{p_{1} \beta_{1} I_{3}^{*} S^{*}+q_{1} \beta_{2} J^{*} S^{*}+\xi_{1} J^{*}}{I_{1}^{*}} \\
b_{2}=\frac{p_{2} \beta_{1} I_{3}^{*} S^{*}+q_{2} \beta_{2} J^{*} S^{*}+\varepsilon_{1} I_{1}^{*}+\xi_{2} J^{*}}{I_{2}^{*}} \\
b_{3}=\frac{p_{3} \beta_{1} I_{3}^{*} S^{*}+q_{3} \beta_{2} J^{*} S^{*}+\varepsilon_{2} I_{2}^{*}+\xi_{3} J^{*}}{I_{3}^{*}} \\
b_{4}=\frac{r I_{3}^{*}}{J^{*}}
\end{gathered}
$$

Substituting the results from (26)-(30) in (25), we get

$$
\begin{aligned}
V_{1}^{\prime}= & \left(1-\frac{S^{*}}{S}\right)\left[\beta_{1} I_{3}^{*} S^{*}+\beta_{2} J^{*} S^{*}+\mu S^{*}-\left(\beta_{1} I_{3} S+\beta_{2} J S+\mu S\right)\right] \\
& +B\left(1-\frac{I_{1}^{*}}{I_{1}}\right)\left[p_{1} \beta_{1} I_{3} S+q_{1} \beta_{2} J S+\xi_{1} J-\frac{p_{1} \beta_{1} I_{3}^{*} S^{*}+q_{1} \beta_{2} J^{*} S^{*}+\xi_{1} J^{*}}{I_{1}^{*}} I_{1}\right] \\
& +C\left(1-\frac{I_{2}^{*}}{I_{2}}\right)\left[p_{2} \beta_{1} I_{3} S+q_{2} \beta_{2} J S+\varepsilon_{1} I_{1}+\xi_{2} J-\frac{p_{2} \beta_{1} I_{3}^{*} S^{*}+q_{2} \beta_{2} J^{*} S^{*}+\varepsilon_{1} I_{1}^{*}+\xi_{2} J^{*}}{I_{2}^{*}} I_{2}\right] \\
& +D\left(1-\frac{I_{3}^{*}}{I_{3}}\right)\left[p_{3} \beta_{1} I_{3} S+q_{3} \beta_{2} J S+\varepsilon_{2} I_{2}+\xi_{3} J-\frac{p_{3} \beta_{1} I_{3}^{*} S^{*}+q_{3} \beta_{2} J^{*} S^{*}+\varepsilon_{2} I_{2}^{*}+\xi_{3} J^{*}}{I_{3}^{*}} I_{3}\right] \\
& \left.+E\left(1-\frac{J^{*}}{J}\right)\left[r I_{3}-\frac{r I_{3}^{*}}{J^{*}}\right]\right]
\end{aligned}
$$

Using the following variables substitutions

$$
\frac{S}{S^{*}}=x, \frac{I_{1}}{I_{1}^{*}}=y, \frac{I_{2}}{I_{2}^{*}}=z, \frac{I_{3}}{I_{3}^{*}}=u, \frac{J}{J^{*}}=v
$$

Equation (31) reduces to

$$
\begin{aligned}
V_{1}^{\prime}= & -\mu S^{*} \frac{(1-x)^{2}}{x}+\left(1-\frac{1}{x}\right)\left[\beta_{1} I_{3}^{*} S^{*}(1-x u)+\beta_{2} J^{*} S^{*}(1-x v)\right] \\
& +B\left(1-\frac{1}{y}\right)\left[p_{1} \beta_{1} I_{3}^{*} S^{*}(x u-y)+q_{1} \beta_{2} J^{*} S^{*}(x v-y)+\xi_{1} J^{*}(v-y)\right] \\
& +C\left(1-\frac{1}{z}\right)\left[p_{2} \beta_{1} I_{3}^{*} S^{*}(x u-z)+q_{2} \beta_{2} J^{*} S^{*}(x v-z)+\varepsilon_{1} I_{1}^{*}(y-z)+\xi_{2} J^{*}(v-z)\right] \\
& +D\left(1-\frac{1}{u}\right)\left[p_{3} \beta_{1} I_{3}^{*} S^{*}(x u-u)+q_{3} \beta_{2} J^{*} S^{*}(x v-u)+\varepsilon_{2} I_{2}^{*}(z-u)+\xi_{3} J^{*}(v-u)\right] \\
& +E\left(1-\frac{1}{v}\right)\left[r I_{3}^{*}(u-v)\right]
\end{aligned}
$$




$$
\begin{aligned}
= & -\mu S^{*} \frac{(1-x)^{2}}{x}+\beta_{1} I_{3}^{*} S^{*}+\beta_{2} J^{*} S^{*}+B p_{1} \beta_{1} I_{3}^{*} S^{*}+B q_{1} \beta_{2} J^{*} S^{*}+B \xi_{1} J^{*}+C p_{2} \beta_{1} I_{3}^{*} S^{*} \\
& +C q_{2} \beta_{2} J^{*} S^{*}+C \varepsilon_{1} I_{1}^{*}+C \xi_{2} J^{*}+D p_{3} \beta_{1} I_{3}^{*} S^{*}+D q_{3} \beta_{2} J^{*} S^{*}+D \varepsilon_{2} I_{2}^{*}+D \xi_{3} J^{*}+E r I_{3}^{*} \\
& +x u\left[-\beta_{1} I_{3}^{*} S^{*}+B p_{1} \beta_{1} I_{3}^{*} S^{*}+C p_{2} \beta_{1} I_{3}^{*} S^{*}+D p_{3} \beta_{1} I_{3}^{*} S^{*}\right] \\
& +x v\left[-\beta_{2} J^{*} S^{*}+B q_{1} \beta_{2} J^{*} S^{*}+C q_{2} \beta_{2} J^{*} S^{*}+D q_{3} \beta_{2} J^{*} S^{*}\right] \\
& +u\left[\beta_{1} I_{3}^{*} S^{*}-D p_{3} \beta_{1} I_{3}^{*} S^{*}-D q_{3} \beta_{2} J^{*} S^{*}-D \varepsilon_{2} I_{2}^{*}-D \xi_{3} J^{*}+E r I_{3}^{*}\right] \\
& +v\left[\beta_{2} J^{*} S^{*}+B \xi_{1} J^{*}+C \xi_{2} J^{*}+D \xi_{3} J^{*}-E r I_{3}^{*}\right]+y\left[-B p_{1} \beta_{1} I_{3}^{*} S^{*}-B q_{1} \beta_{2} J^{*} S^{*}-B \xi_{1} J^{*}+C \varepsilon_{1} I_{1}^{*}\right] \\
& +z\left[-C p_{2} \beta_{1} I_{3}^{*} S^{*}-C q_{2} \beta_{2} J^{*} S^{*}-C \varepsilon_{1} I_{1}^{*}-C \xi_{2} J^{*}+D \varepsilon_{2} I_{2}^{*}\right]-\frac{x u}{y}\left(B p_{1} \beta_{1} I_{3}^{*} S^{*}\right)-\frac{x v}{y}\left(B q_{1} \beta_{2} J^{*} S^{*}\right) \\
& -\frac{x u}{z}\left(C p_{2} \beta_{1} I_{3}^{*} S^{*}\right)-\frac{x v}{z}\left(C q_{2} \beta_{2} J^{*} S^{*}\right)-x\left(D p_{3} \beta_{1} I_{3}^{*} S^{*}\right)-\frac{x v}{u}\left(D q_{3} \beta_{2} J^{*} S^{*}\right)-\frac{v}{y}\left(B \xi_{1} J^{*}\right) \\
& -\frac{y}{z}\left(C \varepsilon_{1} I_{1}^{*}\right)-\frac{v}{z}\left(C \xi_{2} J^{*}\right)-\frac{z}{u}\left(D \varepsilon_{2} I_{2}^{*}\right)-\frac{v}{u}\left(D \xi_{3} J^{*}\right)-\frac{u}{v}\left(E r I_{3}^{*}\right)-\frac{1}{x}\left(\beta_{1} I_{3}^{*} S^{*}+\beta_{2} J^{*} S^{*}\right)
\end{aligned}
$$

The only variable terms that appears in (33) with positive coefficients are $x u, x v, u, v, y$ and $z$. Making the coefficient of $x u, x v, u, v, y$ and $z$ equal to zero, we have

$$
\begin{gathered}
B p_{1}+C p_{2}+D p_{3}-1=0 \\
B q_{1}+C q_{2}+D q_{3}-1=0 \\
\beta_{2} S^{*}+B \xi_{1}+C \xi_{2}+D \xi_{3}-E b_{4}=0 \\
\beta_{1} S^{*}+E r I_{3}^{*}-D b_{3}=0 \\
C \varepsilon_{1}-B b_{1}=0 \\
D \varepsilon_{2}-C b_{2}=0
\end{gathered}
$$

Solving equations (34), (38) and (39), we get

$$
B=\frac{\varepsilon_{1} \varepsilon_{2}}{p_{1} \varepsilon_{1} \varepsilon_{2}+p_{2} b_{1} \varepsilon_{2}+p_{3} b_{1} b_{2}}, C=\frac{b_{1} \varepsilon_{2}}{p_{1} \varepsilon_{1} \varepsilon_{2}+p_{2} b_{1} \varepsilon_{2}+p_{3} b_{1} b_{2}}, D=\frac{b_{1} b_{2}}{p_{1} \varepsilon_{1} \varepsilon_{2}+p_{2} b_{1} \varepsilon_{2}+p_{3} b_{1} b_{2}}
$$

From equations (36) and (37), we have

$$
E=\frac{\beta_{2} S^{*}}{b_{4}}+\frac{\varepsilon_{1} \varepsilon_{2} \xi_{1}+\varepsilon_{2} b_{1} \xi_{2}+b_{1} b_{2} \xi_{3}}{b_{4}\left(p_{1} \varepsilon_{1} \varepsilon_{2}+p_{2} b_{1} \varepsilon_{2}+p_{3} b_{1} b_{2}\right)} \text { and } E=\frac{b_{1} b_{2} b_{3}}{r\left(p_{1} \varepsilon_{1} \varepsilon_{2}+p_{2} b_{1} \varepsilon_{2}+p_{3} b_{1} b_{2}\right)}-\frac{\beta_{1} S^{*}}{r}
$$

Thus, we get

$$
\begin{gathered}
{\left[\frac{\beta_{2} S^{*}}{b_{4}}+\frac{\varepsilon_{1} \varepsilon_{2} \xi_{1}+\varepsilon_{2} b_{1} \xi_{2}+b_{1} b_{2} \xi_{3}}{b_{4}\left(p_{1} \varepsilon_{1} \varepsilon_{2}+p_{2} b_{1} \varepsilon_{2}+p_{3} b_{1} b_{2}\right)}\right]-\left[\frac{b_{1} b_{2} b_{3}}{r\left(p_{1} \varepsilon_{1} \varepsilon_{2}+p_{2} b_{1} \varepsilon_{2}+p_{3} b_{1} b_{2}\right)}-\frac{\beta_{1} S^{*}}{r}\right]} \\
=\frac{\left(\beta_{1} b_{4}+\beta_{2} r\right) S^{*}}{b_{4} r}-\frac{b_{1} b_{2} b_{3} b_{4}+\left(\varepsilon_{1} \varepsilon_{2} \xi_{1}+\varepsilon_{2} b_{1} \xi_{2}+b_{1} b_{2} \xi_{3}\right) r}{b_{4} r\left(p_{1} \varepsilon_{1} \varepsilon_{2}+p_{2} b_{1} \varepsilon_{2}+p_{3} b_{1} b_{2}\right)} \\
=\frac{\left(p_{1} \varepsilon_{1} \varepsilon_{2}+p_{2} b_{1} \varepsilon_{2}+p_{3} b_{1} b_{2}\right)\left(\beta_{1} b_{4}+\beta_{2} r\right) S^{*}-\left[b_{1} b_{2} b_{3} b_{4}+\left(\varepsilon_{1} \varepsilon_{2} \xi_{1}+\varepsilon_{2} b_{1} \xi_{2}+b_{1} b_{2} \xi_{3}\right) r\right]}{b_{4} r\left(p_{1} \varepsilon_{1} \varepsilon_{2}+p_{2} b_{1} \varepsilon_{2}+p_{3} b_{1} b_{2}\right)}
\end{gathered}
$$




$$
\begin{aligned}
& =\frac{\left(b_{1} b_{2} b_{3} b_{4}-b_{1} b_{2} \xi_{3} r-b_{1} \varepsilon_{2} \xi_{2} r-\varepsilon_{1} \varepsilon_{2} \xi_{1} r\right) R_{0} \cdot \frac{\mu}{\Lambda} \frac{\Lambda}{\mu R_{0}}-\left[b_{1} b_{2} b_{3} b_{4}+\left(\varepsilon_{1} \varepsilon_{2} \xi_{1}+\varepsilon_{2} b_{1} \xi_{2}+b_{1} b_{2} \xi_{3}\right) r\right]}{b_{4} r\left(p_{1} \varepsilon_{1} \varepsilon_{2}+p_{2} b_{1} \varepsilon_{2}+p_{3} b_{1} b_{2}\right)} \\
& =0
\end{aligned}
$$

Hence, equation (33) becomes

$$
\begin{aligned}
V_{1}^{\prime}= & \mu S^{*} \frac{(1-x)^{2}}{x}+\beta_{1} I_{3}^{*} S^{*}+\beta_{2} J^{*} S^{*}+B p_{1} \beta_{1} I_{3}^{*} S^{*}+B q_{1} \beta_{2} J^{*} S^{*}+B \xi_{1} J^{*}+C p_{2} \beta_{1} I_{3}^{*} S^{*} \\
& +C q_{2} \beta_{2} J^{*} S^{*}+C \varepsilon_{1} I_{1}^{*}+C \xi_{2} J^{*}+D p_{3} \beta_{1} I_{3}^{*} S^{*}+D q_{3} \beta_{2} J^{*} S^{*}+D \varepsilon_{2} I_{2}^{*}+D \xi_{3} J^{*} \\
& +E r I_{3}^{*}-\frac{x u}{y}\left(B p_{1} \beta_{1} I_{3}^{*} S^{*}\right)-\frac{x v}{y}\left(B q_{1} \beta_{2} J^{*} S^{*}\right)-\frac{x u}{z}\left(C p_{2} \beta_{1} I_{3}^{*} S^{*}\right)-\frac{x v}{z}\left(C q_{2} \beta_{2} J^{*} S^{*}\right) \\
& -x\left(D p_{3} \beta_{1} I_{3}^{*} S^{*}\right)-\frac{x v}{u}\left(D q_{3} \beta_{2} J^{*} S^{*}\right)-\frac{v}{y}\left(B \xi_{1} J^{*}\right)-\frac{y}{z}\left(C \varepsilon_{1} I_{1}^{*}\right)-\frac{v}{z}\left(C \xi_{2} J^{*}\right)-\frac{z}{u}\left(D \varepsilon_{2} I_{2}^{*}\right) \\
& -\frac{v}{u}\left(D \xi_{3} J^{*}\right)-\frac{u}{v}\left(E r I_{3}^{*}\right)-\frac{1}{x}\left(\beta_{1} I_{3}^{*} S^{*}+\beta_{2} J^{*} S^{*}\right)
\end{aligned}
$$

Thus, we have

$$
\begin{aligned}
& V_{1}^{\prime}=-\mu S^{*} \frac{(1-x)^{2}}{x}+\frac{b_{1} b_{2}}{p_{1} \varepsilon_{1} \varepsilon_{2}+p_{2} b_{1} \varepsilon_{2}+p_{3} b_{1} b_{2}} p_{3} \beta_{1} I_{3}^{*} S^{*}\left(2-x-\frac{1}{x}\right) \\
&+\frac{b_{1} b_{2}}{p_{1} \varepsilon_{1} \varepsilon_{2}+p_{2} b_{1} \varepsilon_{2}+p_{3} b_{1} b_{2}} \xi_{3} J^{*}\left(2-\frac{v}{u}-\frac{u}{v}\right)+\frac{b_{1} \varepsilon_{2}}{p_{1} \varepsilon_{1} \varepsilon_{2}+p_{2} b_{1} \varepsilon_{2}+p_{3} b_{1} b_{2}} \xi_{2} J^{*}\left(3-\frac{v}{z}-\frac{z}{u}-\frac{u}{v}\right) \\
&+ \frac{b_{1} \varepsilon_{2}}{p_{1} \varepsilon_{1} \varepsilon_{2}+p_{2} b_{1} \varepsilon_{2}+p_{3} b_{1} b_{2}} p_{2} \beta_{1} I_{3}^{*} S^{*}\left(3-\frac{1}{x}-\frac{z}{u}-\frac{x u}{z}\right) \\
&+\frac{b_{1} b_{2}}{p_{1} \varepsilon_{1} \varepsilon_{2}+p_{2} b_{1} \varepsilon_{2}+p_{3} b_{1} b_{2}} q_{3} \beta_{2} J^{*} S^{*}\left(3-\frac{1}{x}-\frac{u}{v}-\frac{x v}{u}\right) \\
&+\frac{\varepsilon_{1} \varepsilon_{2}}{p_{1} \varepsilon_{1} \varepsilon_{2}+p_{2} b_{1} \varepsilon_{2}+p_{3} b_{1} b_{2}} p_{1} \beta_{1} I_{3}^{*} S^{*}\left(4-\frac{1}{x}-\frac{z}{u}-\frac{y}{z}-\frac{x u}{y}\right) \\
&+\frac{\varepsilon_{1} \varepsilon_{2}}{p_{1} \varepsilon_{1} \varepsilon_{2}+p_{2} b_{1} \varepsilon_{2}+p_{3} b_{1} b_{2}} q_{1} \beta_{2} J^{*} S^{*}\left(5-\frac{1}{x}-\frac{z}{u}-\frac{u}{v}-\frac{y}{z}-\frac{x v}{y}\right) \\
&+\frac{b_{1} \varepsilon_{2}}{p_{1} \varepsilon_{1} \varepsilon_{2}+p_{2} b_{1} \varepsilon_{2}+p_{3} b_{1} b_{2}} q_{2} \beta_{2} J^{*} S^{*}\left(4-\frac{1}{x}-\frac{z}{u}-\frac{u}{v}-\frac{x v}{z}\right) \\
&+\frac{\varepsilon_{1} \varepsilon_{2}}{p_{1} \varepsilon_{1} \varepsilon_{2}+p_{2} b_{1} \varepsilon_{2}+p_{3} b_{1} b_{2}} \xi_{1} J^{*}\left(4-\frac{v}{y}-\frac{y}{z}-\frac{u}{v}-\frac{z}{u}\right)
\end{aligned}
$$

Since the arithmetic mean is greater than or equal to the geometric mean, we have

(i) $2-x-\frac{1}{x} \leq 0$ for $x>0$ and $2-x-\frac{1}{x}=0$ if and only if $x=1$;

(ii) $2-\frac{v}{u}-\frac{u}{v} \leq 0$ for $u, v>0$ and $2-\frac{v}{u}-\frac{u}{v}=0$ if and only if $u=v$;

(iii) $3-\frac{v}{z}-\frac{z}{u}-\frac{u}{v} \leq 0$ for $u, v, z>0$ and $3-\frac{v}{z}-\frac{z}{u}-\frac{u}{v}=0$ if and only if $u=v=z$;

(iv) $3-\frac{1}{x}-\frac{z}{u}-\frac{x u}{z} \leq 0$ for $x, u, z>0$ and $3-\frac{1}{x}-\frac{z}{u}-\frac{x u}{z}=0$ if and only if $x=1, u=z$; 
(v) $3-\frac{1}{x}-\frac{u}{v}-\frac{x v}{u} \leq 0$ for $x, u, v>0$ and $3-\frac{1}{x}-\frac{u}{v}-\frac{x v}{u}=0$ if and only if $x=1, u=v$;

(vi) $4-\frac{1}{x}-\frac{z}{u}-\frac{y}{z}-\frac{x u}{y} \leq 0$ for $x, y, z, u>0$ and $4-\frac{1}{x}-\frac{z}{u}-\frac{y}{z}-\frac{x u}{y}=0$ if and only if $x=1, y=z=u$;

(vii) $5-\frac{1}{x}-\frac{z}{u}-\frac{u}{v}-\frac{y}{z}-\frac{x v}{y} \leq 0$ for $x, y, z, u, v>0$ and $5-\frac{1}{x}-\frac{z}{u}-\frac{u}{v}-\frac{y}{z}-\frac{x v}{y}=0$ if and only if $x=1, y=z=u=v$;

(viii) $4-\frac{1}{x}-\frac{z}{u}-\frac{u}{v}-\frac{x v}{z} \leq 0$ for $x, z, u, v>0$ and $4-\frac{1}{x}-\frac{z}{u}-\frac{u}{v}-\frac{x v}{z}=0$ if and only if $x=1, u=v=z$;

(ix) $4-\frac{v}{y}-\frac{y}{z}-\frac{u}{v}-\frac{z}{u} \leq 0$ for $y, z, u, v>0$ and $4-\frac{v}{y}-\frac{y}{z}-\frac{u}{v}-\frac{z}{u}=0$ if and only if $y=z=u=v$;

Therefore, $V_{1}^{\prime} \leq 0$ for $x, y, z, u, v>0$ and $V_{1}^{\prime}=0$ if and only if $x=1, y=z=u=v$. The maximum invariant set of system (7)-(12) on the set $\left\{(x, y, z, u, v): V_{1}^{\prime}=0\right\}$ is the singleton $(1,1,1,1,1)$. Thus for system (7)-(12), the endemic equilibrium $E^{*}$ is globally asymptotically stable if $p_{1}=q_{1}, p_{2}=q_{2}, p_{3}=q_{3}$ and $R_{0}>1$ by LaSalle [23].

\section{Numerical Simulation}

To validate the analytical results established in previous section, we conduct the numerical simulation by taking an example. The system (7)-(12) is simulated by fixing the default values of the parameters as

$$
\begin{gathered}
\Lambda=0.55, \beta_{1}=0.0001, \beta_{2}=0.006, \varepsilon_{1}=0.002, \varepsilon_{2}=0.003, \\
r=0.01, \rho=0.03, \mu=0.01, \alpha=0.01 .
\end{gathered}
$$

For the other parameters chosen as

$$
p_{1}=0.5, p_{2}=0.3, p_{3}=0.2 \text {, }
$$

$q_{1}=0.4, q_{2}=0.3, q_{3}=0.3, \xi_{1}=0.4, \xi_{2}=0.2$ and $\xi_{3}=0.4$, figs. 2(a)-2(b) demonstrate that the reproduction number $R_{0}=0.18041<1$ which indicates that the disease free equilibrium points $E_{0}$ is globally stable.

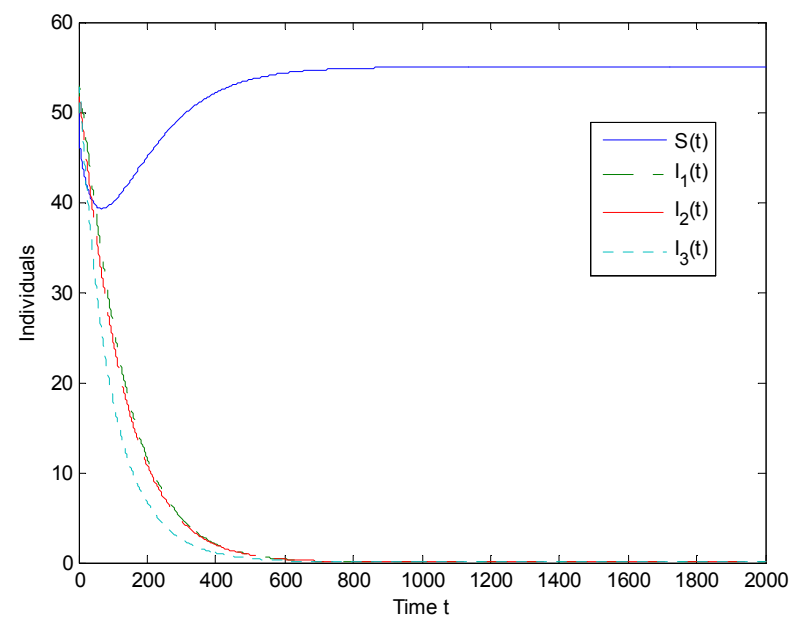

2(a)

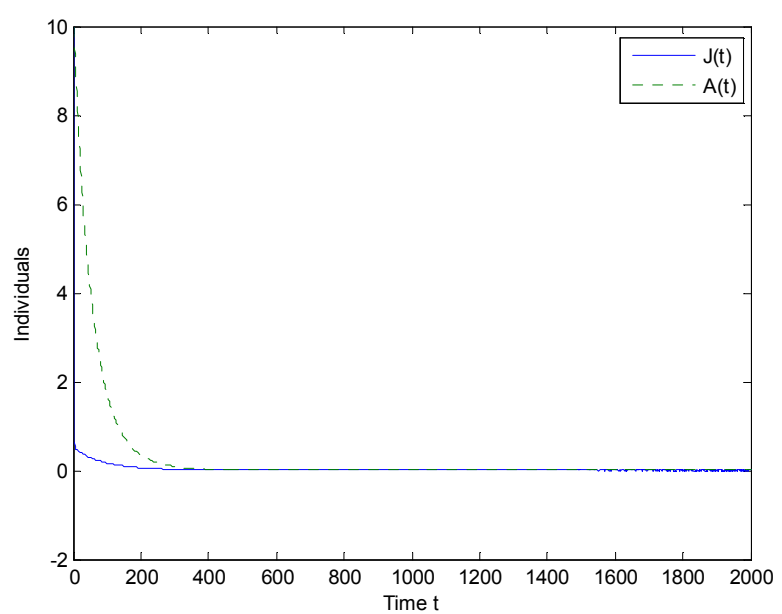

2(b)

Figs. 2. Stability of the disease free equilibrium point $E_{0}$

For the figs. 3(a)-3(b), the parameters are set as $p_{1}=q_{1}=0.05, p_{2}=q_{2}=0.9, \quad p_{3}=q_{3}=0.05$, $\xi_{1}=0.005, \xi_{2}=0.001$ and $\xi_{3}=0.003$. It is clear from these figs. that the reproduction number $R_{0}=1.0191>1$ which corresponds to the global stability of endemic equilibrium points $E^{*}$.

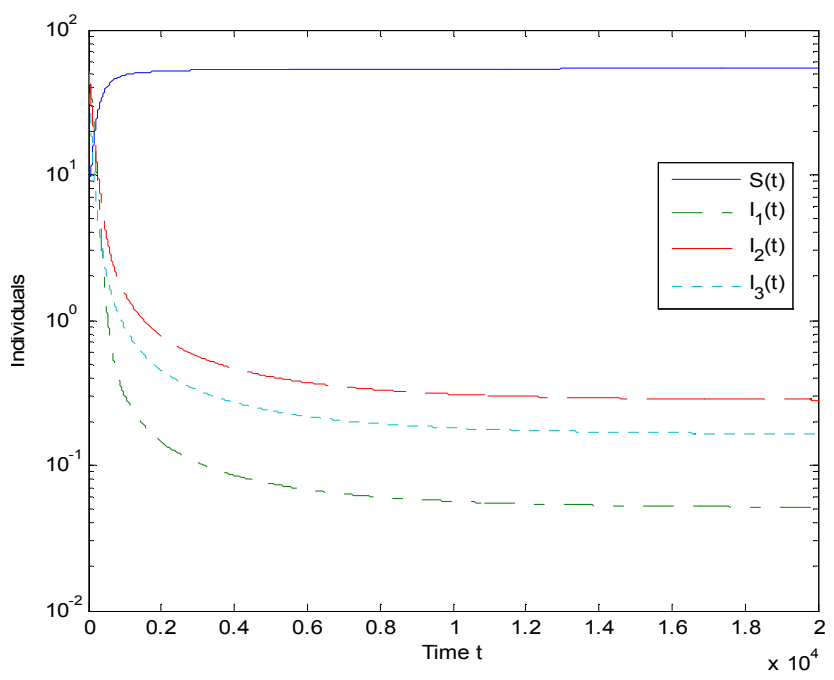

3(a) 


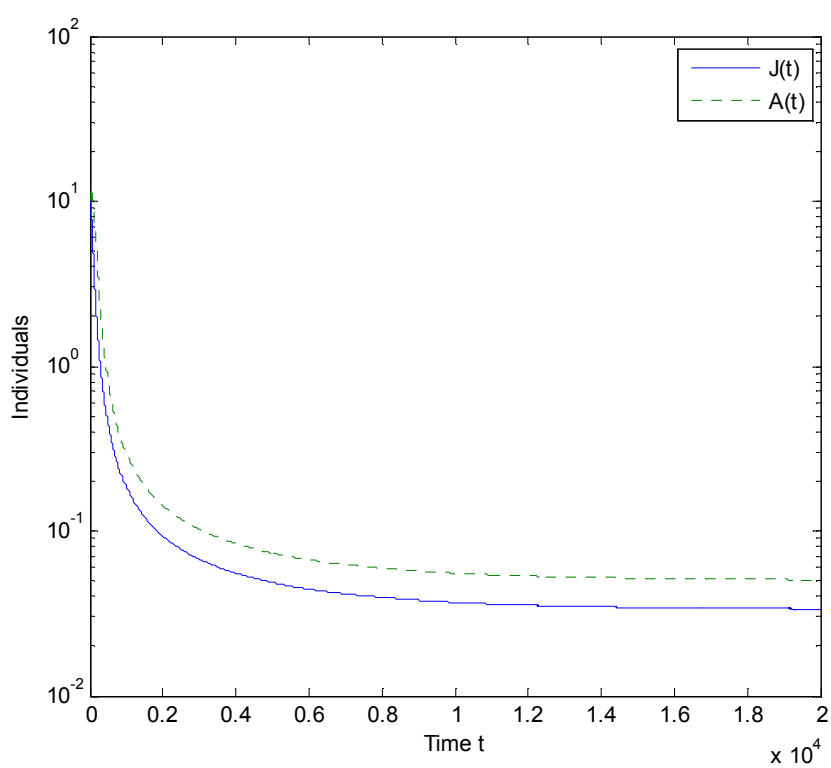

$3(b)$

Figs. 3. Stability of the endemic equilibrium point $E^{*}$.

Figs. 4(a)-4(b) depict the relation among (a) $R_{0} \& \xi_{1}, \xi_{2}$ and (b) $R_{0} \& \xi_{2}, \xi_{3}$, respectively. From these figs., it is clear that when $\xi_{1}, \xi_{2}$ are small and $\xi_{3}$ is too much large then the basic reproduction number $R_{0}$ of the system (7)-(12) is less than unity. Biologically, we can infer that the treatment for individuals in slow, medium and fast compartments has significant impact on the transmission dynamic of the disease.

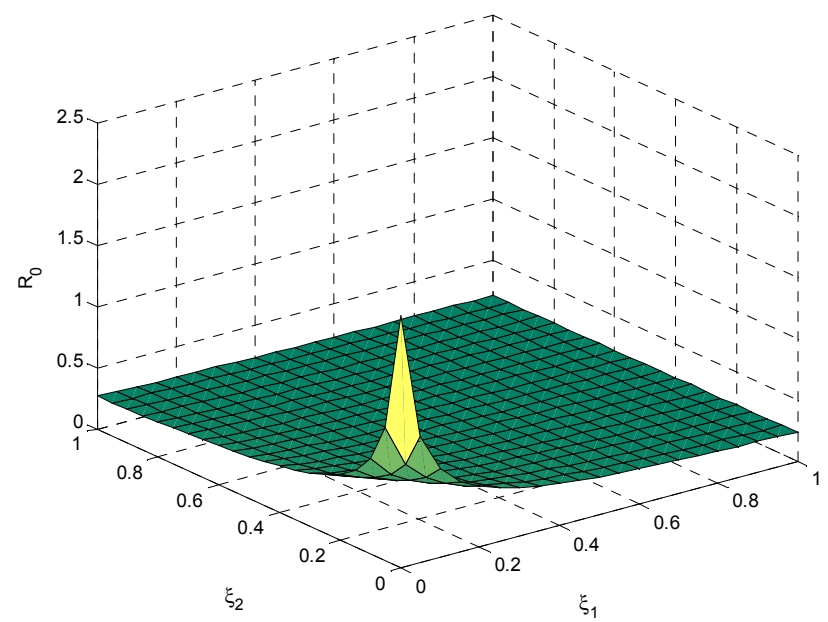

4(a)

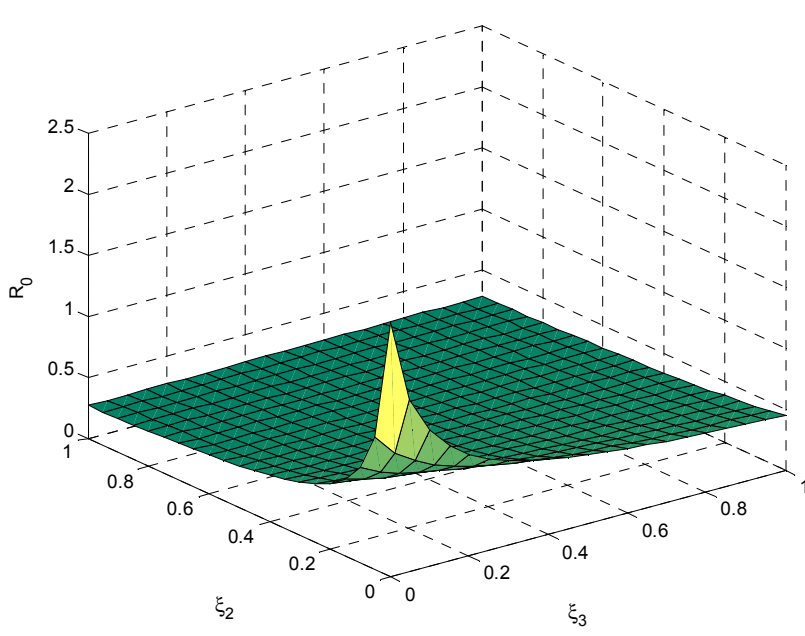

4(b)

Figs. 4. Relationship among $R_{0}(a) \xi_{1}$ and $\xi_{2}$, (b) $\xi_{2}$ and $\xi_{3}$

Fig. 5 depicts the relationship among $R_{1}, \xi_{1}$ and $\xi_{2}$. It is seen that when the values of $\xi_{1}$ and $\xi_{2}$ are less than $R_{1}$ of the model for slow and fast compartments, our results are in good agreement with the results of Huo and Feng [10] for two latent compartments model for which the basic reproduction number $\left(\mathrm{R}_{1}\right)$ is given by

$$
R_{1}=\frac{\left[\beta_{1} b_{3}\left(\varepsilon p+b_{1}(1-p)\right)+\beta_{2} p_{1}\left(\varepsilon q+b_{1}(1-q)\right)\right] \frac{\Lambda}{\mu}}{b_{1} b_{2} b_{3}-\left(\varepsilon \xi_{1}+b_{1} \xi_{2}\right) p_{1}}
$$

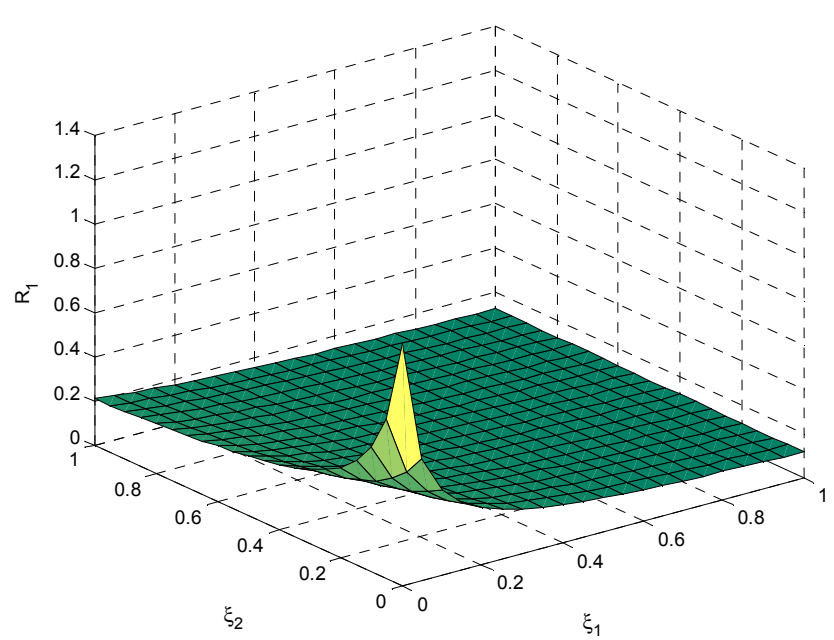

Fig. 5. Relationship among $R_{1}, \xi_{1}$ and $\xi_{2}$ 


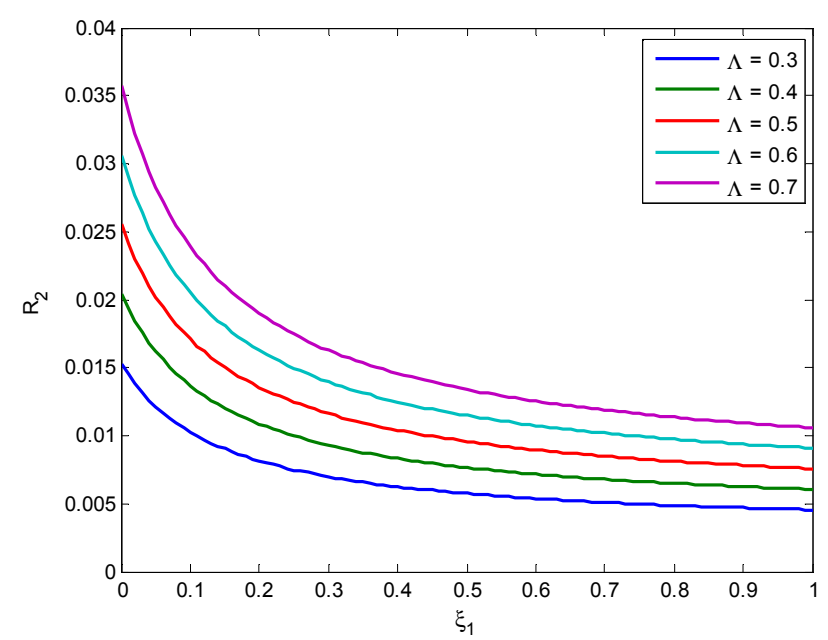

$6(a)$

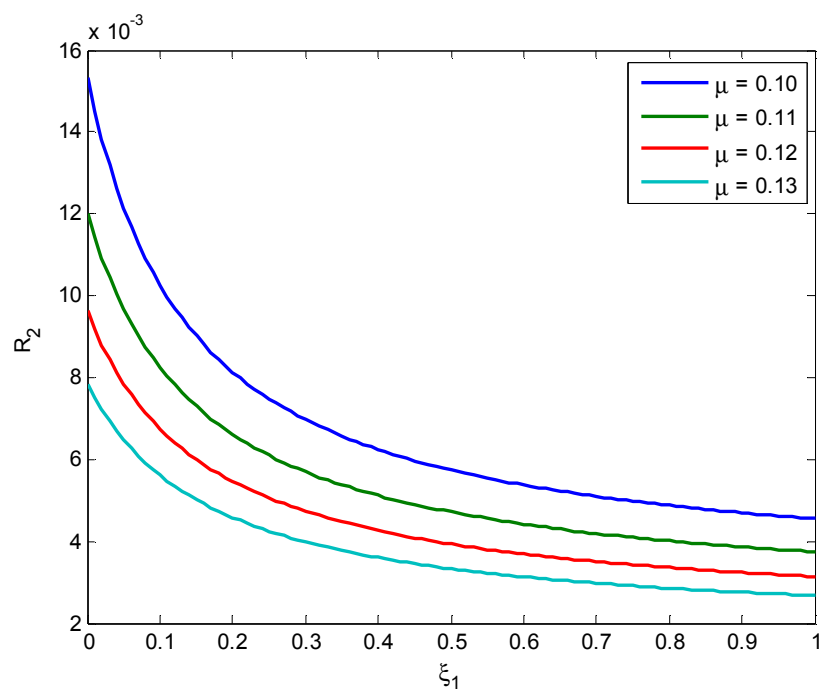

$6(b)$

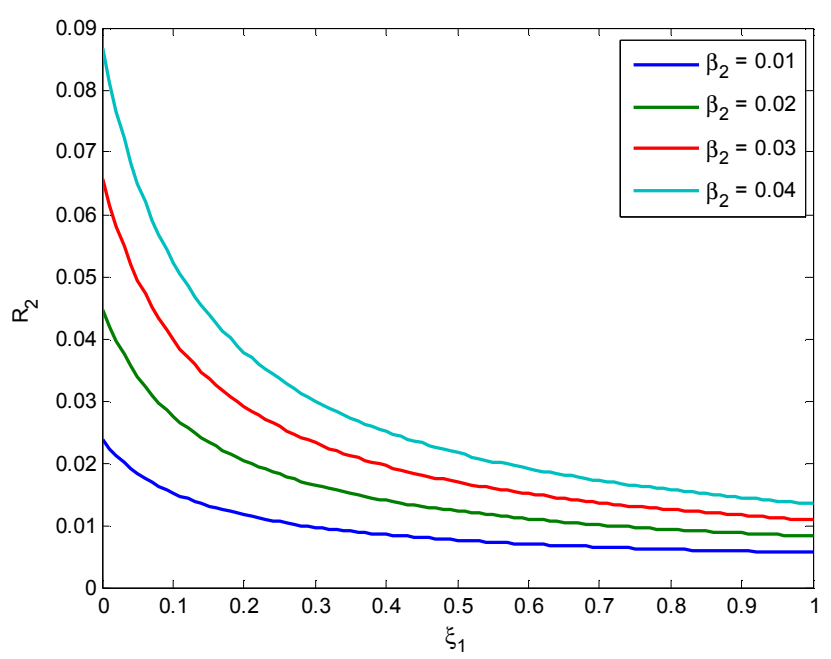

6(c)

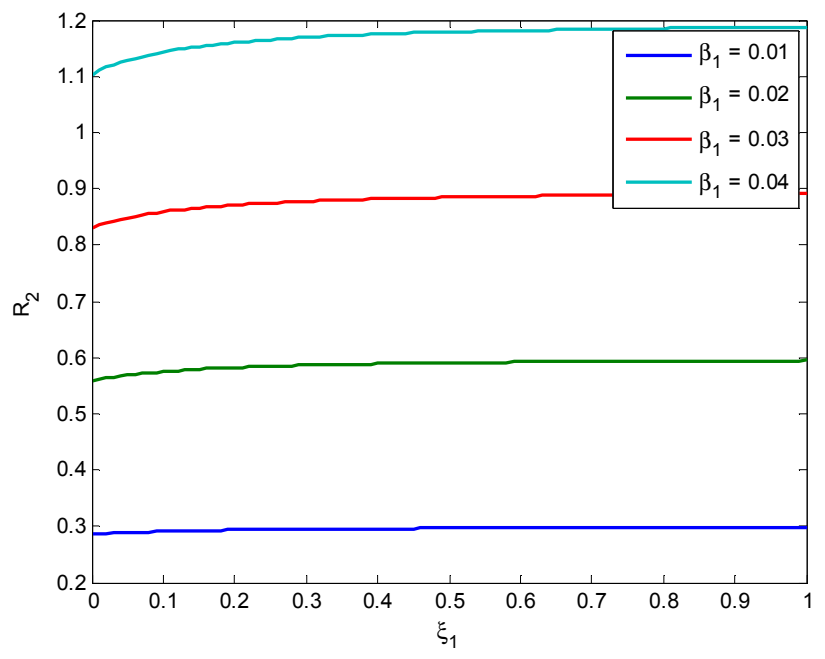

$6(\mathrm{~d})$

Figs. 6. Relationship among $R_{2}$ and $\xi_{1}$ for different values of (a) $\Lambda$ (b) $\mu$ (c) $\beta_{1}(d) \beta_{2}$

For varying values of parameters $\Lambda, \beta_{1}, \beta_{2}$ and $\mu$, respectively, figs. $6(\mathrm{a})-6(\mathrm{~d})$ exhibit the relation between $R_{2}=\frac{\left[\beta_{1}\left(\mu+p_{2}+\xi_{1}\right)+\beta_{2} p_{1}\right] \frac{\Lambda}{\mu}}{\left(\mu+p_{1}\right)\left(\mu+p_{2}\right)+\mu \xi_{1}}$ and $\xi_{1}$. The other parameters are set as $\Lambda=0.3, \mu=0.1$, $\beta_{1}=0.0001, \beta_{2}=0.006, p_{1}=0.01$ and $p_{2}=0.03$. Fig. 6(a) displays that $R_{2}$ increases when the value of $\Lambda$ goes on increasing but it decreases as $\xi_{1}$ increases. In fig. 6(b) reveals the trends of $R_{2}$ with $\xi_{1}$ for different values of $\mu$. It is observed that the value of $R_{2}$ decreases with the increment in $\mu$. Fig. 6(c) shows that $R_{2}$ increases by increasing $\beta_{2}$. Biologically it can be interpreted easily that the transmission coefficient of symptomatic stage has significant effect on $R_{2}$ for single latent stage. Fig. 6(d) reveals that the value of $R_{2}$ remains almost constant when the value of $\beta_{1}$ increases; this demonstrates that the transmission coefficient of fast latent compartment has also significant effect on $R_{2}$ for single latent stage.

\section{Conclusion}

In this paper, the proposed epidemic model has three latent stages to explore the dynamical behavior of HIV/AIDS. Based on numerical simulation, it is concluded that the disease free equilibrium is both locally and globally asymptotically stable whenever the basic reproduction number is less than unity. Further, the endemic equilibrium is globally asymptotically stable whenever the corresponding reproduction number is greater than unity. It is noticed that the treatment of infective compartment has positive impact on HIV/AIDS control. The present model can be extended to multi-latent compartments for infective population depending 
upon several stages due to treatment and socio-economic constraints.

\section{References}

[1] May R. M. and Anderson R. M., "Transmission dynamics of HIV infection", Nature. 236, 137-142 (1987).

[2] Anderson R. M., Medly G. F., May R. M. and Johnson A. M., "A preliminary study of the transmission dynamics of the human immunodeficiency virus (HIV), the causative agent of AIDS”, IMA J. Math. Appl. Med. Biol. 3, 229-263 (1986).

[3] Anderson R. M., "The role of mathematical models in the study of HIV transmission and the epidemiology of AIDS", J. AIDS. 1, 241-256 (1988).

[4] Morgan D., Mahe C., Mayanja B., Okongo J. M., Lubega R. and Whitworth J. A., "HIV-linfection in rural Africa: is there a difference in median time to AIDS and survival compared with that in industrialized countries", AIDS. 16, 597-632 (2002).

[5] Bachar M. and Dorfmayr A., "HIV treatment models with time delay", C. R. Biol. 327, 983-994 (2004).

[6] Stoddart C. A. and Reyes R. A., "Models of HIV-1 disease: A review of current status, Drug Discov", Today: Disease Models. 3, 113-119 (2006).

[7] Alexander M. E. and Moghadas S. M., "Bifurcation analysis of an SIRS epidemic model with generalised incidence", SIAM J. Appl. Math. 65, 1794-1816 (2005).

[8] Cai L., Li X. and Ghosh M., "Global stability of a stagestructured epidemic model with a nonlinear incidence", Appl. Math. Comput. 214, 73-82 (2009).

[9] Cai L., Li X., Ghosh M. and Guo B., "Stability analysis of an HIV/AIDS epidemic model with treatment", J. Comput. Appl. Math. 229, 313-323 (2009).

[10] Huo H.F. and Feng L.X., "Global stability for an HIV/AIDS epidemic model with different latent stages and treatment", Appl. Math. Model. 37, 1480-1489 (2013).

[11] Okosun K. O., Makinde O. D. and Takaidza I., "Impact of optimal control on the treatment of HIV/AIDS and screening of unaware infectives", Appl. Math. Model. 37, 3802-3820 (2013).
[12] Defeng L. and Wang B., "A novel time delayed HIV/AIDS model with vaccination \& antiretroviral therapy and its stability analysis”, Appl. Math. Model. 37, 4608-4625 (2013).

[13] Bhunu C. P. and Mushayabasa S., "Modelling the transmission dynamics of HIV/AIDS and hepatitis $\mathrm{C}$ virus coinfection", HIV/AIDS Rev. 12(2), 37-42 (2013).

[14] Cai L., Guo S. and Wang S., "Analysis of an extended HIV/AIDS epidemic model with treatment", Appl. Math. Comput. 336, 621-627 (2014).

[15] Kaur N., Ghosh M. and Bhatia S. S., "Mathematical analysis of the transmission dynamics of HIV/AIDS: Role of female sex workers”, Appl. Math. Inf. Sci. 8 (5), 2491-2501 (2014).

[16] Elaiw A. M. and Almuallem N. A., "Global properties of delayed-HIV dynamics models with differential drug efficacy in cocirculating target cells", Appl. Math. Comput. 265, 10671089 (2015).

[17] Wang J., Zhang R. and Kuniya T., "Global dynamics for a class of age-infection HIV models with nonlinear infection rate", Math. Analysis and Appl. 432 (1), 289-313 (2015).

[18] Shen M., Xiao Y. and Rong L., "Global stability of an infection-age structured HIV-1 model linking within-host and between-host dynamics", Math. Biosci. 263, 37-50 (2015).

[19] Huo H. F., Chen R. and Wang X. Y., "Modelling and stability of HIV/AIDS epidemic model with treatment", Appl. Math. Model. 40(13-14), 6550-6559 (2016).

[20] Ven den Driessche P. and Watmough J., "Reproduction numbers and sub-threshold endemic equilibria for compartmental models of disease transmission", Math. Biosci. 180, 29-48 (2002).

[21] Lakshmikantham V., Leela S. and Martynyuk A. A., "Stability Analysis of Nonlinear Systems", Marcel Dekker Inc. New York (1989).

[22] Mushayabasa S., Tchuenche J. M., Bhunu C. P. and GwasiraNgarakana E., "Modelling gonorrhoea and HIV cointeraction”, Bio. Systems. 103 (1), 27-37 (2011).

[23] LaSalle J. P., "The stability of dynamical systems", Regional Conference Series in Appl. Math. SIAM. Philadelphia (1976). 